Case Report

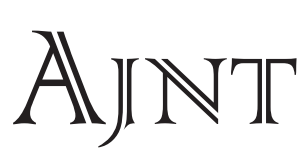

\title{
Reversible Posterior Leukoencephalopathy Syndrome Preceding End Stage Renal Failure as the Initial Presentation of Connective Tissue Disease
}

\author{
Elzubier A Elzubier*
}

Associate nephrologist, renal unit, Colchester University Hospital, Colchester, UK

\begin{abstract}
Introduction: Reversible posterior leukoencephalopathy syndrome (RPLS) is a rare clinico-radiological entity that has been infrequently reported in patients with connective tissue diseases, particularly in those with established renal involvement.
\end{abstract}

Case report: A 51 year old female presented with headache, confusion, transient loss of vision and seizures. She was found to have elevated blood pressure. Her level of consciousness deteriorated rapidly necessitating mechanical ventilation. Hematological and biochemical investigations were essentially normal. Brain CT scan revealed widespread subcortical white matter changes which were more prominent in the posterior parietal lobes, raising the possibility of RPLS. Vasculitic screen revealed elevated ANA, anti-Ro and anti-La antibody titers. Her blood pressure was controlled with medications and this was followed by improvement in her neurological condition. Two weeks later, she had a brain MRI scan which was essentially normal, confirming the diagnosis of RPLS. Her renal function, however, deteriorated rapidly during her hospital stay and a renal biopsy showed features consistent with the microangiopathic hemolytic uremic syndrome. She progressed to end stage renal failure and was maintained on regular dialysis. Eight months later she successfully received a kidney transplant.

Conclusion: RPLS can be the initial presentation in patients with connective tissue diseases, and this possibility should not be overlooked by the physician.

\footnotetext{
* Corresponding author; Colchester University Hospital, NHS Foundation Trust, Turner Road, Colchester, Essex, CO4 5JL, UK.

E mail: Elzubier@aol.com
}

Keywords: connective tissue disease, renal failure, reversible posterior leukoencephalopathy, RPLES

\section{Introduction}

Neurological symptoms and signs are common in patients with connective tissue disease, and can be the presenting feature of these conditions. Reversible posterior leukoenchephalopathy syndrome (RPLS) is a rare clinicoradiological entity that has been infrequently reported in patients with connective tissue disease, particularly those with established renal impairment [1-4]. Here we describe a patient with a connective tissue disease, probably Sjogren's syndrome, who initially presented with typical features of RPLS prior to the development of end stage renal failure,

\section{Case report}

A 51 years old female was admitted to the emergency medical unit with two days history of headache, neck pain, confusion, blurring of vision and episodes of transient loss of vision. She had past medical history of migraine and Raynaud's phenomenon.

On examination she was confused with a Glasgow Coma Scale (GCS) of 12/15, her pulse rate was $88 \mathrm{bpm}$ in sinus rhythm, blood pressure was $200 / 100 \mathrm{mmHg}$, and temperature was $37.1^{\circ} \mathrm{C}$. Systemic examination including full neurological examination was unremarkable. In particular, examination of the visual fields and the optic fundi showed no residual defects, indicating that her episodes of blindness were probably cortical in origin. There were no signs of meningeal irritation.

Initial investigations revealed total white cell count of $8400 / \mu 1$, hemoglobin $13.8 \mathrm{~g} / \mathrm{dl}$, platelets $212000 / \mu \mathrm{l}$ and normal differential counts. Her serum creatinine was $1.1 \mathrm{mg} / \mathrm{dl}$, urea $34.2 \mathrm{mg} / \mathrm{dl}$, CRP $11 \mathrm{mg} / 1$ (normal range $0-10$ ). Her liver function tests were normal and a mid-stream urine sample showed no abnormalities. 
Figure 1: Non- contrast CT of the brain showing wide-spread areas of hypodensity in both parietal and occipital lobes that are more marked on the right side (a). A repeat CT scan of the brain after six months showing complete resolution of the white matter changes (b)

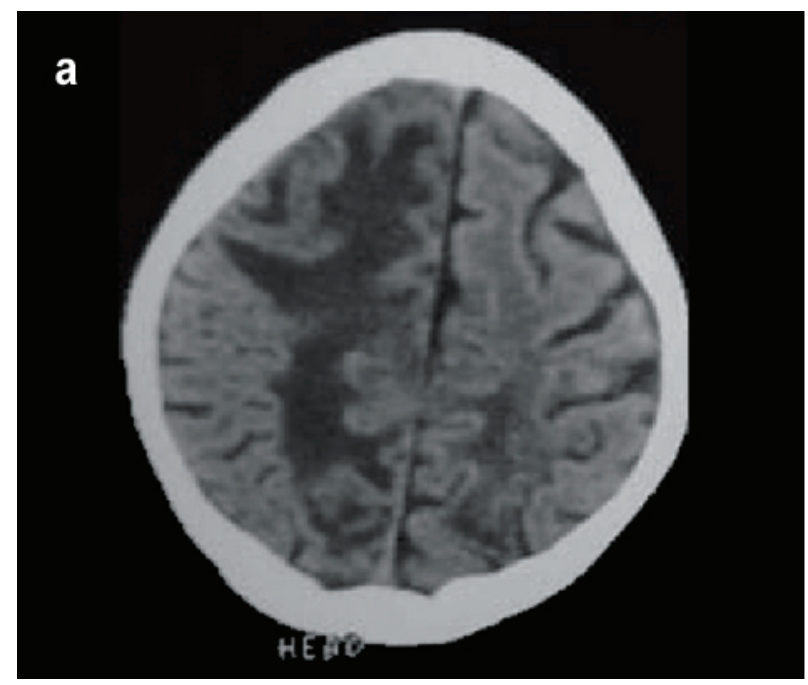

Provisional differential diagnoses at that stage were sub-arachnoid hemorrhage (SAH) and meningoencephalitis. While she was waiting to have CT head scan, she developed a generalised tonic seizure and her GCS dropped to $7 / 15$. She was transferred to the Intensive Therapy Unit (ITU) and put on mechanical ventilation. The CT head scan was obtained. It showed widespread subcortical white matter changes which were more prominent in the posterior parietal lobes with no other abnormalities detected (Figure 1). The radiological differential diagnoses included RPLS, vasculitis, acute demyelinating encephalomyelitis and infective encephalitis. Cerebro-spinal fluid examination obtained by lumber puncture was not diagnostic. Vasculitic screen showed a positive ANA titer of $1 / 320$ with speckled and nucleolar pattern on immunoflorecence. Anti DNA antibodies titer was $4 \mathrm{IU} / \mathrm{ml}$ (normal level $0-20 \mathrm{IU} / \mathrm{ml}$ ). Both anti Ro and anti La antibodies were positive, but antibodies against the rest of extractable nuclear antigens were negative. Caticholamines level in 24 hour urine collection was normal.

While in the ITC the blood pressure was controlled with glyceryl trinitrate (GTN) infusion. She stayed in ITU for $48 \mathrm{hrs}$ and was then discharged to the medical ward. Her blood pressure was then controlled with atenolol and nifedipine.

One day after discharge from ITU her renal function rapidly deteriorated and she became oliguric. Ultrasound scan of the kidneys and renal tract was reported as essentially normal. Complement and immunoglobulin levels were normal. Subsequently, a renal biopsy was performed and was reported to show features consistent

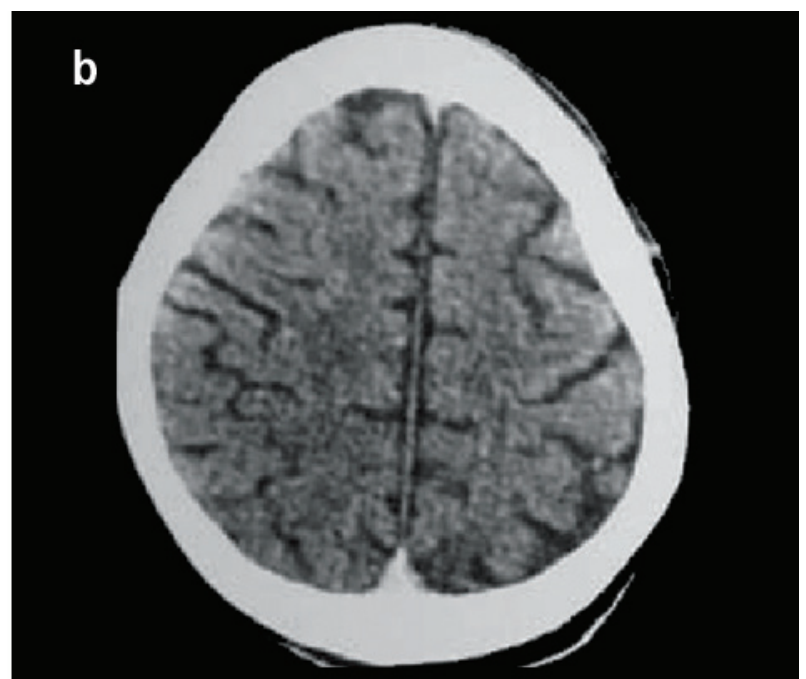

with microangiopathic, hemolytic uremic syndrome. In view of the positive serology suggestive of Sjogren's syndrome, she had a labial biopsy which showed no features supportive of this diagnosis.

She was commenced on dialysis. Her neurological signs improved. An MRI scan of the brain performed two weeks later was entirely normal, in keeping with the diagnosis of reversible posterior leukoencephalopathy syndrome. Another CT scan of the brain obtained six months later was also normal (Figure1). However, she remained dialysis dependant until she had a successful renal transplant eight months later.

\section{Discussion}

Reversible posterior leukoencephalopathy syndrome (RPLS) refers to a clinico-radiological entity characterized by headache, confusion, visual disturbances, seizures and posterior transient changes on neuro-imaging. Hinchey et al described this condition in 1996 and suggested that RPLS represented a localized manifestation of hypertensive encephalopathy occurring secondary to hypertensive crisis [1].

RPLS has been described in several cases of systemic lupus erythematosus [2-4]. Other associated conditions include pre eclampsia, thrombotic thrombocytopenic purpura and hemolytic uremic syndrome as well as drug toxicity from agents such as cyclosporine, tacrolimus, cisplatin, acyclovir and erythropoietin stimulating agents. The association with acyclovir is of particular importance as this is the main differential diagnosis of worsening viral encephalitis. 
The role of neuro-imaging is to establish the diagnosis and to exclude other neurological conditions. Classical CT findings are those of bilaterally symmetric low attenuation in the posterior, parietal and occipital lobes, whereas MR imaging demonstrates hyper-intensity on T2-weighted images in the same distribution [5]. Differential diagnosis of RPLS includes posterior circulation infarcts, venous thrombosis, demyelinating disease, vasculitis, and encephalitis. Since RPLS is often unsuspected by clinicians, radiologist maybe the first to suggest the diagnosis.

Two pathophysiological mechanisms for RPLS have been proposed. One postulates cerebral vasospasm with resulting ischemia within the involved territories, whereas the second postulation is a breakdown in cerebrovascular auto-regulation with ensuing interstitial extravasation of fluid. The posterior circulation supplied by vertibrobasilar system has poor sympathetic innervation and, therefore, is frequently involved.

\section{Conclusion}

RPLS can be the initial presentation in patients with connective tissue disease, and this possibility should always be kept in mind.

\section{References}

1. Hinchey J, Chaves C, Appignani B, Breen J, Pao L, Wang A, Pessin MS, Lamy C, Mas JL, Caplan LR. A reversible posterior leukoencephalopathy syndrome. N Engl J Med. 1996 Feb 22;334(8):494-500.

2. Leroux G, Sellam J, Costedoat-Chalumeau N, Le Thi Huong D, Combes A, Tieulié N, Haroche J, Amoura Z, Nieszkowska A, Chastre J, Dormont D, Piette JC. Posterior reversible encephalopathy syndrome during systemic lupus erythematosus: four new cases and review of the literature. Lupus. 2008;17(2):139-47.

3. Min L, Zwerling J, Ocava LC, Chen IH, Putterman C. Reversible posterior leukoencephalopathy in connective tissue diseases. Semin Arthritis Rheum. 2006 Jun;35(6):388-95.

4. El Karoui K, Le Quintrec M, Dekeyser E, Servais A, Hummel A, Fadel F, Fakhouri F. Posterior reversible encephalopathy syndrome in systemic lupus erythematosus. Nephrol Dial Transplant. 2008 Feb;23(2):757-63

5. Covarrubias DJ, Luetmer PH, Campeau NG. Posterior reversible encephalopathy syndrome: prognostic utility of quantitative diffusion-weighted MR images. AJNR Am J Neuroradiol. 2002 Jun-Jul;23(6):1038-48. 